\title{
Rheumatoide Arthritis: Verschiedene Tapering- Strategien auf dem Prüfstand
}

van Mulligen E et al. Tapering towards DMARD-

free remission in established rheumatoid

arthritis: 2-year results of the TARA trial. Ann

Rheum Dis 2020; 79: 1174-1181. doi: 10.1136/

annrheumdis-2020-217485

Dank der modernen Pharmakotherapie erreichen viele Patienten mit einer rheumatoiden Arthritis (RA) eine anhaltende Remission. In diesem Fall können die Medikamente langsam ausgeschlichen werden. Ziel ist dabei die dauerhafte Beschwerdefreiheit ohne DMARDs (disease modifying antirheumatic drugs). In welcher Reihenfolge sollte das Tapering der verschiedenen Wirkstoffe optimalerweise erfolgen? Und wie häufig gelingt eine DMARD-freie Remission?

Diese und andere Fragen untersuchten niederländische Wissenschaftler im Rahmen der an 12 niederländischen Kliniken durchgeführten randomisierten TARA-Studie. An der Untersuchung nahmen 189 erwachsene RA-Patienten teil, welche unter einer Kombination aus konventionellen synthetischen DMARDs (meist Methotrexat) und einem TNF (Tumornekrosefaktor)-Inhibitor über mindestens 3 Monate eine niedrige Krankheitsaktivität (Disease Activity Score/ DAS44 $\leq 2,4$ und Swollen Joint Count/SJC $\leq 1$ ) aufwiesen. Rund eine Hälfte der Studienteilnehmer reduzierte über einen Zeitraum von 12 Monaten zunächst schrittweise die Dosis der konventionellen DMARDs, im zweiten Jahr dann die Dosis des TNF-Inhibitors. Bei der anderen Hälfte der Patienten erfolgte das Tapering in der umgekehrten Reihenfolge. Zu Studienbeginn wurden die Patienten gebeten, keine Glukokortikoide anzuwenden. Nichtsteroidale Antiphlogistika sowie intraartikuläre Glukokortikoidinjektionen waren aber während der Studienteilnahme erlaubt. Als primären Studienendpunkt definierten die Forscher die Häufigkeit von Erkrankungsschüben innerhalb des 2-jährigen Nachbeobachtungszeitraums. Ferner prüften sie, wie viele Studienteilnehmer eine DMARD-freie Remission erreichten. Weitere Endpunkte bildeten unter anderem die Krankheitsaktivität, die funk- 
tionelle Leistungsfähigkeit und der radiologische Krankheitsprogress.

\section{Ergebnisse}

Die mediane Symptomdauer betrug im Studienkollektiv (66\% Frauen, Durchschnittsalter 56,6 Jahre) 6,2 Jahre. Eine Remission (DAS44<1,6) gelang bei $80 \%$ der Patienten, die zuerst die konventionellen synthetischen DMARDs ausschlichen und bei $88 \%$ der Patienten, mit initialer Dosisreduktion des TNF-Inhibitors. Die kumulative Schubrate betrug nach 24 Monaten in diesen beiden Gruppen 61 bzw. 62 \% ( $p=0,84)$. Eine DMARD-freie Remission gelang nach 2 Jahren bei insgesamt $15 \%$ der Studienteilnehmer, wobei diejenigen Patienten, welche zuerst die konventionellen synthetischen DMARDs ausschlichen, diesbezüglich leicht im Vorteil waren (20 vs. $11 \%$; $=0,07$ ). Die Reihenfolge der Dosisdeeskalation hatte weder einen signifikanten Einfluss auf die Krankheitsaktivität noch die funktionelle Leistungsfähigkeit der Patienten oder den radiologischen Krankheitsprogress.

\section{FAZIT}

Nur ein relativ geringer Anteil der RA-Patienten erreicht durch das Tapering eine DMARD-freie Remission, schließen die Forscher. Die Reihenfolge der DMARD- bzw. TNF-InhibitorDeeskalation scheint dabei unter klinischen Aspekten keine wesentliche Rolle zu spielen. Bei der Wahl der Tapering-Strategie könnten ihrer Einschätzung nach daher finanzielle Aspekte den Ausschlag geben: Aus ökonomischer Sicht wäre es sinnvoll, die teureren TNF-Inhibitoren zuerst auszuschleichen.

Dr. med. Judith Lorenz, Künzell 